Original Research Paper

\title{
Integration of Artificial Neural Network and Expert System for Material Classification of Natural Fibre Reinforced Polymer Composites
}

\author{
${ }^{1}$ Basheer Ahmed Ahmed Ali, \\ ${ }^{1-3}$ Mohd Sapuan Salit, ${ }^{2,3}$ Edi Syams Zainudin and ${ }^{4}$ Mohamed Othman \\ ${ }^{I}$ Institute of Advanced Technology, \\ ${ }^{2}$ Department of Mechanical and Manufacturing Engineering, \\ ${ }^{3}$ Institute of Tropical Forestry and Forest Products, \\ ${ }^{4}$ Faculty of Computer Science and Information Technology, \\ Universiti Putra Malaysia, 43400 UPM Serdang, Selangor, Malaysia
}

Article history

Received: 25-11-2014

Revised: $12-02-2015$

Accepted: 03-04-2015

\section{Corresponding Author:}

Mohd Sapuan Salit

Department of Mechanical and

Manufacturing Engineering,

Universiti Putra Malaysia,

43400 UPM Serdang, Selangor,

Malaysia

Tel: $+603-89466318$

Fax: $+603-86567122$

Email: sapuan@upm.edu.my

\begin{abstract}
Diversified choice of materials from natural fibre reinforced polymer composites with similar properties complicate the materials selection for engineering products. Implementation of expert system alone makes it difficult to scrutinize the vast selected materials. Hybrid of expert system with neural network technology is desired. Classification of material through neural network under various criteria influences the decision in narrowing down the selection. In this study, the integration of artificial neural network with expert system for material classification is explored. The computational tool Matlab is proposed for classification and the materials focused were natural fibre composites. Levenberg-Marquardt training algorithm, which provides faster rate of convergence, is applied for training the feed forward network. The system proves to be consistant with 93.3\% classification accuracy with 15 neurons in the hidden layer. The validation of the output is compared with the target on the basis of desired mechanical properties of natural fibre reinforced polymer composites for automotive interior components.
\end{abstract}

Keywords: Artificial Neural Network, Expert System, Material Classification, Natural Fibre Composites, Feed Forward Network

\section{Introduction}

The innovation in material science and technology reveals more materials than ever before and the selection menu become countless for the engineers. Ashby (2004) described the available materials for the engineers are vast and expected to something over 120,000 materials of choice. Materials selection is an important criterion for engineering applications. The explosion all over the world is increasingly using the computing power to solve a complex engineering problem that offers optimum solution. Artificial Neural Network (ANN) was a powerful computational model that simulates the neurons of a biological nervous system. It consists of a set of connected cells called neurons. The neurons receive impluses as input and perform transformation of input information and transmit to output neurons (Yegnanarayana, 2009). It deals with non-linear problems for an accurate analytical solution. It can learn to detect complex patterns in data by training and thus classifying it while testing with real data. ANN provides practical solution for pattern recognition, classification and optimization problems. It is also used in signal processing, speech recognition, condition monitoring and functional approximation.

Somkuwar et al. (2011) used expert network with integration of radial basis neural network architecture for materials selection. Sensorial properties and cost of steel metal were considered for selection. They developed the expert system with visual $\mathrm{c}++$ and integrated with neural network tool box of Matlab. Sapuan and Mujtaba (2010) developed a neural network based materials selection system for selecting optimal material from natural fibre polymer composites. They implemented back propagation algorithm to correlate between the inputs and to predict the output materials. Further the predicted output was processed using multi-attribute ranking 
method to select most suitable material. Golmohammadi (2011) described a model for decision making by implementing artifical neural network for fuzzy multicriteria decision making problems. The technique of pairwise comparison from analytical hierarcy process was applied to rank the criterion and feed forward method was used to train the network. The model was demonstrated in detail for suppliers' performation in product evaluation. Goel and Chen (1996) demonstrated the application of neuro-expert network for materials selection. They utilized neural network connectionist approach with back propagation for training data samples. The expert system evaluated the output of neural network on the basis of price, availability in stock and closeness to desired material properties. Fazilat et al. (2012) proposed a new method for predicting the mechanical properties by Artificial Neural Networks (ANNs) and adaptive Neuro-Fuzzy Inference System (ANFIS) in polymer composites. They made brief instruction to the theoretical background and constructed ANNs and ANFIS models and employed them for analyzing the results with 16 data sets. They claimed that the obtained results were well adopted with minimum errors.

Smith et al. (2002) found that neural network approach was suitable for powder metallurgy modelling for materials selection purposes. Multiple inputs, modelling of highly non-linear responses and the avoidance of noise effects had been provided by training a back propagation neural network with experimental data. The neural network deductions for process inputs were compared to those generated by regression analysis. Charniya and Dudul (2011) developed a prototype for classification of material type and surface roughness. An optimum Multilayer Perceptron Neural Network (MLPNN) model was designed using digital signal processing tools to measure the bouncing plunger probe on the plain surface. The signal generated from optical mouse was recorded on a PC and a three layer neural network classifer with 19 neurons in the hidden layer- 1 and 20 neurons in the hidden layer- 2 was found to be optimal for training the network with conjugategradient learning algorithm. Thus neural network classifier consistently performed with $97.5 \%$ accuracy for non-linear classification.

Zhou et al. (2009) presented their approach for multiobjective materials selection in sustainable products. They implemented artificial neural network with genetic algorithm for optimizing the multi-objectives of materials and selecting the best candidate materials. They took into consideration not only the mechanical and economical properties (Sapuan and Abdalla, 1998), but also the environmental factors for selection. However, to the knowledge of the authors, limited studies have been conducted on materials selection of natural fibre reinforced polymer composites with ANN.

Natural Fibre Composite (NFC) is an emerging alternative material for petro-chemical material to enforce the green technology (Ahmed Ali et al., 2013) and this sustainable material is the dire need of this globe to protect environmental consciousness. It has a potential market segment and significantly penetrates all the applications and mainly in automotive industry (Mohanty et al., 2005). The percentage of natural fibre reinforced composite in the market segment is shown in Fig 1. The properties of NFC significantly change depending on the climate and environmental conditions and it was hard to predict the values as it goes with different fibre loadings (Sapuan et al., 2003). Therefore prediction of output using ANN alone would not produce a worthy result.

The above literature review inspires the incorporation of rule based expert system with ANN technology for material classification. In this study we focus on few properties of natural fibre composite used as an alternative material for synthetic materials in the interior component of automobile. The objective of this research is to integrate the ANN with the expert system output to classify the natural fibre composite materials in accordance with the range specified in product design specification.

\section{Methodology}

The proposed system in this research comprises an expert system integrated with a neural network for material classification. The present research is an elaboration of the authors' previous research work on rule based expert system which was applied for optimum selection of natural fibre composite materials (Ahmed Ali et al., 2013). This materials selection procedure accommodates two steps of approach with three modules; the first by implementing the Java based expert system through the programming tool NetBean, to identify those candidate materials that contain the essential attributes meeting the required range specified in product design specifications. The second is to apply the technique of artificial neural network concept through the computational Matlab tool, to classify the short listed materials against certain other desirable preferences to obtain a final working list of candidate materials. The third module is to use database system software MySQL to accumulate data and to store them as knowledge base in the system. The block diagram of the total system is shown in Fig. 2.

\section{Expert Neural Classifier}

Expert neural classifier executes high efficient learning for faster convergence. In this study, the output data of the expert system was mapped to the input layer of neural network for further classification of the selected materials. The artificial neural network module comprises a fully connected three layer feed forward network with a supervised training algorithm as the target is specified. 


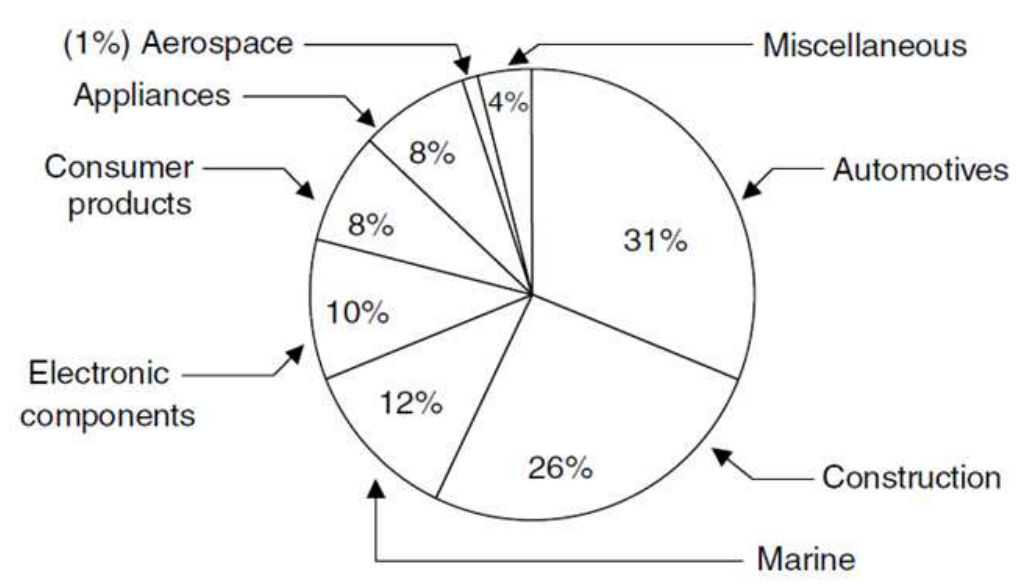

Fig. 1. Natural fibre composite usage in industries (Mohanty et al., 2005)

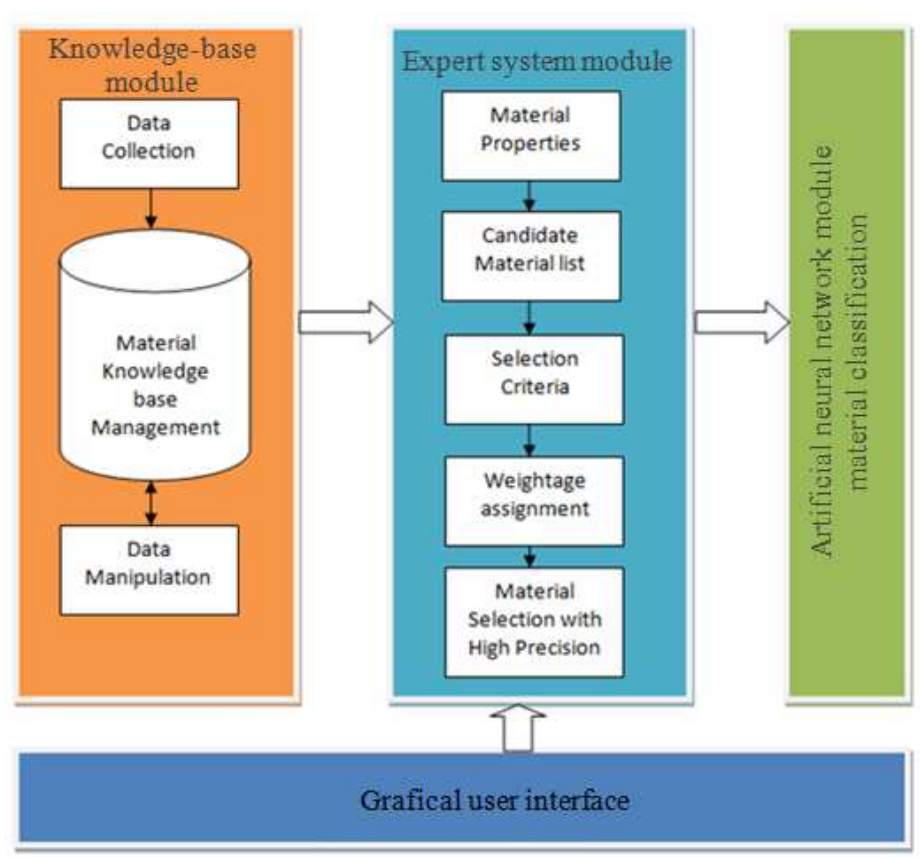

Fig. 2. Block diagram of the expert neural classifier system

The input layer consists of number of neurons equal to the attributes of candidate materials for classification; for this network three attributes (density, tensile strength and Young's modulus) are fed as the parameters for input layer. The target of the network was focused on the value defined by product design specification for automotive interior components (Ahmed Ali et al., 2013). The outputs of the network would be the classification results. The flowchart in Fig. 3 clearly demonstrates the flow control in the Matlab programming with R2010b version using the toolbox of neural network. The network started with initialisation and data extraction from the expert system. The below mentioned command was used to import data by creating a file:

$$
\begin{aligned}
& \text { fid = fopen('private/materialdata.csv'); } \\
& C=\text { textscan (fid, } \% \text { of\%f\%f\%s',' 'delimiter',','); \% Import } \\
& \text { data fclose(fid) }
\end{aligned}
$$

After importing the data to the file, the input data and target data had to be segregated and preprocessed in the form of matrices that can be used with the neural network. The next step was to create a feed forward network. The above input and target data were used to create the network with a unique number of hidden nodes. The syntax was shown as below:

$$
\text { net }=\text { newff(inputs, target,no. of hidden layers })
$$




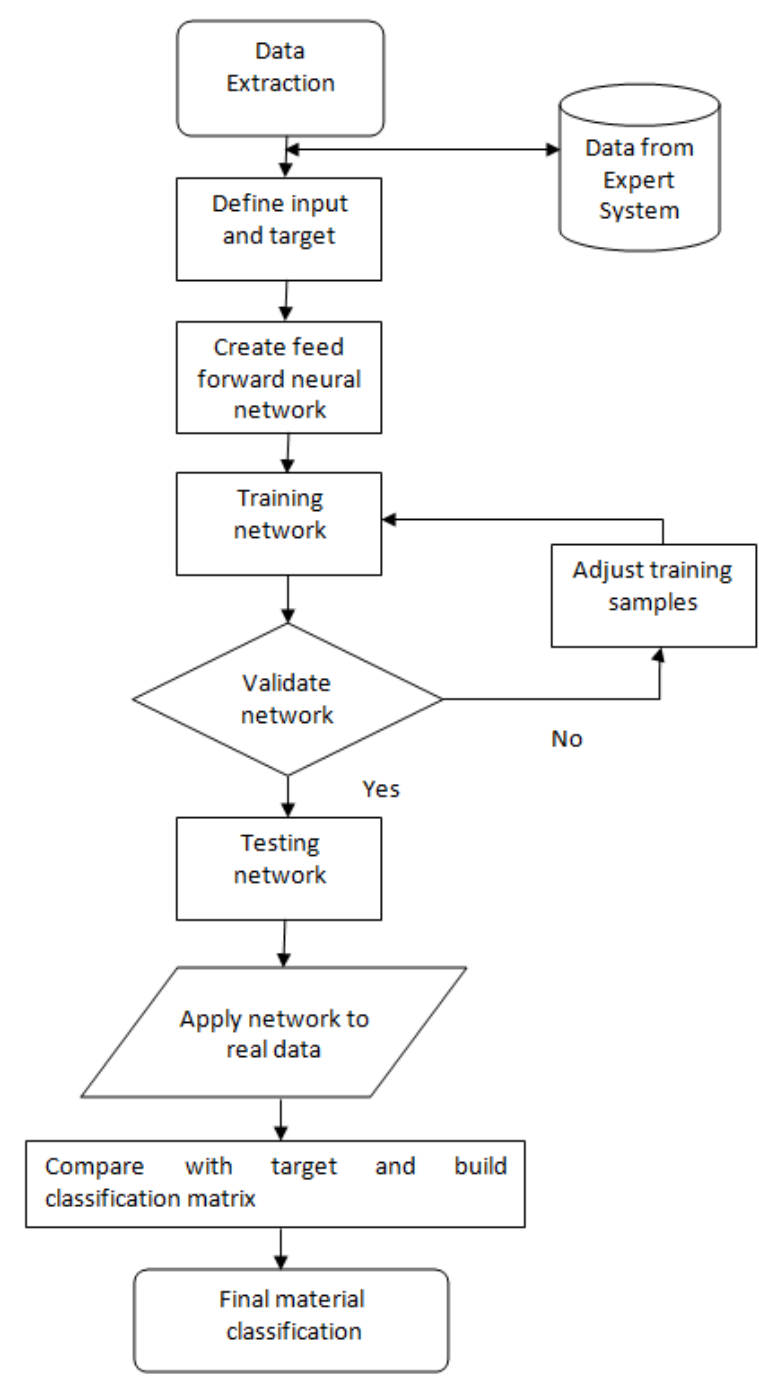

Fig. 3. Expert neural classifier flowchart

There are many criteria deciding the number of hidden layer to create a feed forward network and they are discussed in the next section elobrately. The next important step in the procedure of neural network was training the network with the input and target data. The training continued until the validation of the network was reached. The command to train the network is shown below:

$$
\text { [net,tr] }=\operatorname{train}(\text { net, inputs, target) }
$$

The trained network was then tested with test samples. Next step was to apply the real data for the above constructed network and to record the output. Finally, the output was compared with the target and to build a classification matrix.

\section{Knowledge Base Management System (KBMS)}

In the expert neural classifier system the KBMS was the module to store the knowlegde with higher level of information that was represented in the easy manner to manipulate and store the material informations. Access to the knowledge base was performed through the expert system and was transparent to the user. The data manipulation was made easier with add, delete, update, save and search functions. The data required for ANN process were thereby extracted from knowledge base module which were retrieved from the expert system. The data set consists of three entities; density, tensile strength and Young's modulus of the candidate materials. In total of 120 data set of various natural fibre composites were used to train, validate and test the neural network system. Unlike other database, the NFC data are not much matured when compared with metals, alloys and plastics. As the research trends are newly focused on NFC material, it was difficult to retrieve the entire database of NFC from single source. Therefore, for our research purpose the data set were collected from books, published journals and the previous work of principal author and his co-workers (Sapuan et al., 2011). The sample model of data set was shown in Table 1.

\section{Feed Forward Algorithm}

The feed forward neural network is also known as Multi-Layer Perceptrons (MLP) and it is most widely used model of neural network. Where the connections between the units do not form a directed cycle then it is referred to as a feed forward neural network. In feed forward neural network the connections are between two successive layers and the information flows in one direction, forward from the input layer to the output layer via the hidden layers. There are no cycles or loops in this network and no lateral connection between the neurons of the same layer (Sumathi and Surekha, 2010). The structure of 3 layers feed forward neural network with input layers, hidden layers and output layers are shown in Fig. 4.

\section{Levenberg-Marquardt Algorithm}

The Levenberg-Marquardt Algorithm (LMA) is an iterative technique to provide a numerical solution for minimizing a non-linear function. It is an optimization technique to solve large scale non-linear least square problems. The role of LMA behaves in between the Guass-Newton Algorithm (GNA) and Gradient descent method. When the current solution is far from a local minimum and the result $\lambda$ is large, the algorithm behaves like a steepest descent method; slow, but guaranteed to converge. When the current solution is close to a local minimum with small value of result $\lambda$, it becomes a Gauss-Newton method and exhibits fast convergence (Lourakis and Argyros, 2005). 


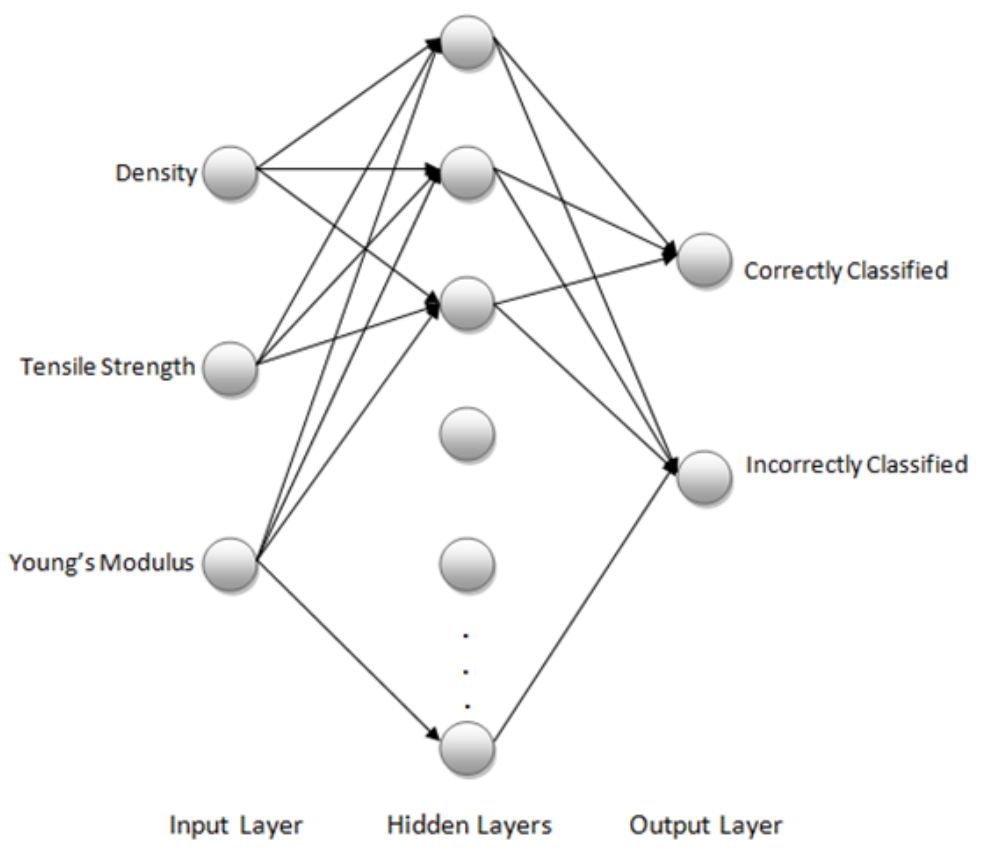

Fig. 4. A 3 layer feed forward neural network

Table 1. Model data set of natural fibre composites

\begin{tabular}{|c|c|c|c|}
\hline Natural fiber composites & Density $\left(\mathrm{kg} / \mathrm{m}^{3}\right)$ & Young's modulus (GPa) & Tensile strength (MPa) \\
\hline $\mathrm{EFB}+\mathrm{PP}$ & 875 & 0.900 & 18.00 \\
\hline Hemp $40 \%+$ PP & 1076 & 6.800 & 52.00 \\
\hline Jute $40 \%+P P$ & 1036 & 3.700 & 28.00 \\
\hline Kenaf $30 \%+P P$ & 1027 & 3.800 & 27.00 \\
\hline Kenaf $40 \%+P P$ & 1072 & 6.800 & 29.00 \\
\hline Kenaf $50 \%+P P$ & 1120 & 7.500 & 35.00 \\
\hline Flax $36 \%+$ epoxy & 1250 & 10.890 & 88.30 \\
\hline Flax $36 \%+$ PE & 1250 & 8.350 & 71.60 \\
\hline Oil palm Empty Fruit Bunch (EFB) 13\% + epoxy resin & 1148 & 2.557 & 12.12 \\
\hline Oil palm Empty Fruit Bunch (EFB) $30 \%+$ polyurethane & 1000 & 0.500 & 17.00 \\
\hline Pseudo-stem banana + epoxy resin & 1280 & 1.890 & 45.57 \\
\hline Sisal $30 \%+P P$ & 1032 & 3.800 & 30.00 \\
\hline Sisal 40\% + PP & 1044 & 5.500 & 34.00 \\
\hline Sisal + PVC & 1284 & 4.200 & 10.00 \\
\hline $\mathrm{EFB}+\mathrm{PVC}$ & 1085 & 1.500 & 38.76 \\
\hline Bagasse + PVC & 1465 & 0.700 & 27.00 \\
\hline Kenaf + PVC & 1420 & 1.770 & 46.00 \\
\hline Vetiver grass $20 \%+\mathrm{PP}$ & 991 & 1.500 & 28.00 \\
\hline Vetiver grass (powder) $20 \%+\mathrm{PP}$ & 991 & 1.250 & 22.00 \\
\hline sisal + PE & 1124 & 3.080 & 38.00 \\
\hline flax + PE & 1250 & 8.350 & 71.60 \\
\hline $\mathrm{EFB}+\mathrm{PE}$ & 1086 & 0.520 & 13.00 \\
\hline Sugar palm + epoxy & 1260 & 1.080 & 32.00 \\
\hline
\end{tabular}

\section{Network Training}

The neural network learns through an iterative process and adjusts the weight accordingly to train the network. In the network training procedure initially the network becomes more knowledgeable regarding the problem statement after several iteration of training. The objective of training is to end up with desired output for all possible values of input feed to the network and also to minimise the error function.

\section{Trainlm}

The trainlm is a training function of the network that updates weight and bias values according to Levenberg-Marquardt algorithm. In Matlab the LMA 
is implemented by the function trainlm. The training parameters of trainlm are epochs, time, min_grad, max_fail, mu, mu_dec, mu_inc, mu_max and mem reduc.

Validation parameters are used to stop the training vectors and to decide the performance of the network. In the neural network the mu controls how much the weights are changed on each iteration. It can be as low as $10^{-6}$ and as high as 0.1 . If the mu value is too small then it is expected that the network would converge too slowly. On the other hand if the value of mu is too large then it would cause the network to converge erratically and the network will exhibit chaotic condition (Somkuwar et al., 2010). The parameter mu is multiplied by mu_dec by each iteration. If mu becomes larger than mu_max, the algorithm is stopped.

The training stops when any one of these condition triggers:

- The maximum number of epochs (repetitions) reached

- The maximum time limit exceeds

- Performance is minimized to the goal

- The gradient falls below min_grad

- Mu exceeds mu_max

- Validation check has reached max_fail

\section{Mean Squared Error (MSE)}

The common error function in the neural network to be minimized was the Mean Squared Error (MSE). It is a network performance function that measures the network performance accordingly to minimize the sum of mean squared errors. Like the perceptron learning rule, the mse is an example of supervised training. The network has an input and corresponding target output. As each input is applied to the network, the network output is compared to the target. The error is calculated as the difference between the target output and the network output. The performace of the network is calculated by average of the sum of these errors. The goal of training the netwrok was to minimize the mse.

\section{Results}

Great concern should be given in selecting number of hidden layers and the number of neurons in the hidden layer, as it can have a significant effort on the performance of the network. It is difficult to determine the number of hidden layers for particular learning process. These parameters are usually defined by trail and error method. However, it has been observed in general one hidden layer is sufficient in most ANN applications (Tan et al., 2011; Trzaska and Dobrzanski, 2006: Smith, 1997). So, for this network we decided to have one hidden layer. To decide the number of nodes in the hidden layer, few experiments are conducted ranging from 10 to 30 hidden nodes to study the reliability and performance (mean squared error) of the network. The performance value for different number of hidden nodes with the total number of epochs (repitition) and gradient values are shown in Table 2.

The above values are plotted in graphical representation for better understanding as shown in Fig. 5. It was observed that the artifical neural network with 15 nodes in hidden layers has the least performance value of 0.0353 with total number of 9 epochs and minimum gradient decent of 0.0121 was significantly efficient for this network. It can also be noticed that the network with 25 hidden nodes has the lesser performance value then 15 hidden nodes. Even though the performance value for 25 hidden nodes is 0.0259 , the gradient value in bold denotes 0.0736 which is significantly higher then 15 nodes gradient value. Further analysis was carried out with network performance convergence graph to decide the number of hidden nodes for the network. When the network training performance was plotted with 25 nodes it had exposed a vast difference in the network convergence. While the network with 15 hidden nodes had significantly fast network convergence as shown in Fig. 9.

Similarly, the regression values are plotted for $10,15,20,25$ and 30 hidden nodes as shown in Table 3 to further study the behaviour of these different hidden nodes. It can be observed that 15 hidden nodes have uniform data with regular interval. So, for this work the ANN with one hidden layer and 15 hidden nodes was selected to be efficient with favourable good result.

Therefore, for the current research work for classification of materials using neural network it was decided to have three input layers, one hidden layer with 15 nodes and two output layer. The architecture of the neural network is shown in Fig. 6.

After deciding the neural network architecture with definite structure the network is ready to be trained. The candidate materials were automatically divided into three distinct sets as training, validation and test sets. The training process would use largest set of data and teaches the network to learn the patterns present in the data set. Training continues until the network gradually improves on the validation checks. A final check on the performance of the network is made and the training stops on reaching the maximum validation checks. On completion of the training, the trained network would be tested with the testing samples. The test data provides a complete measure of network accuracy and garantees that the network can be applied to real world data for classification. 


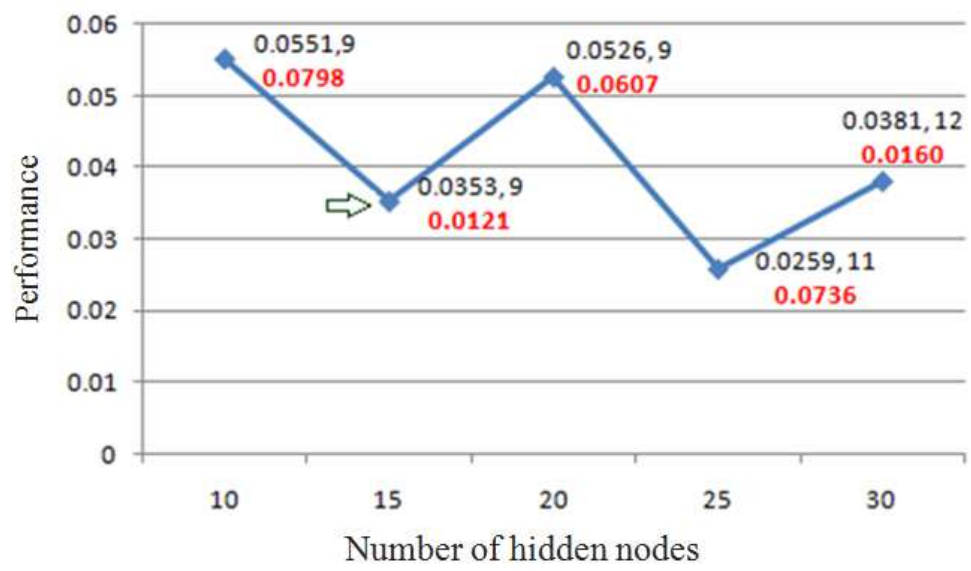

Fig. 5. Performance value verses number of hidden nodes

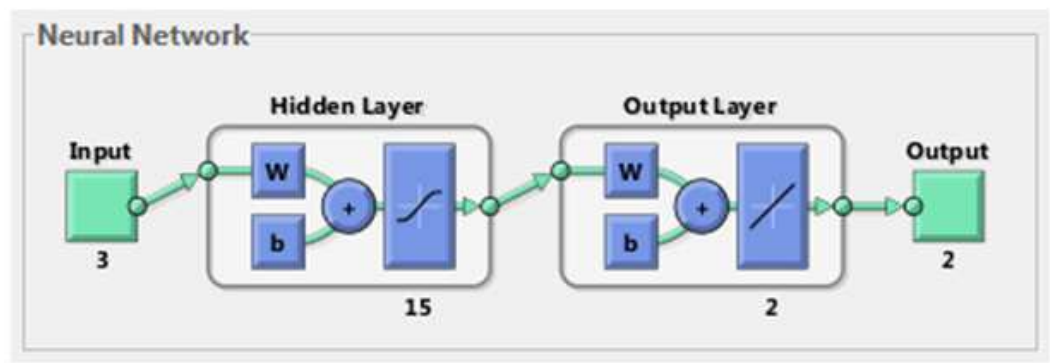

Fig. 6. Neural network architecture

Table 2. Performance of neural network for different number of hidden nodes

\begin{tabular}{llll}
\hline Number of hidden nodes & Total number of Epoch & Performance & Gradient \\
\hline 10 & 9 epochs & 0.0551 & 0.0798 \\
15 & 9 epochs & 0.0353 & 0.0121 \\
20 & 9 epochs & 0.0526 & 0.0607 \\
25 & 11 epochs & 0.0259 & 0.0736 \\
30 & 12 epochs & 0.0381 & 0.0160 \\
\hline
\end{tabular}

Table 3. Regression values of the neural network

\begin{tabular}{lllll}
\hline Number of hidden nodes & Training & Validation & Testing & Overall \\
\hline 10 & 0.86523 & 0.99416 & 0.86445 & 0.88321 \\
15 & 0.89655 & 0.85545 & 0.88918 & 0.88944 \\
20 & 0.81713 & 0.91956 & 0.98757 & 0.85463 \\
25 & 0.89679 & 0.95905 & 0.81018 & 0.89385 \\
30 & 0.90251 & 0.86696 & 0.96329 & 0.90517 \\
\hline
\end{tabular}

\section{Discussion}

The network was executed with the set of knowledge base data of candidate materials from the expert system. Figure 7 shows the over all progress of the network. It clearly indicates that the training stops on reaching the maximum validation checks of 6 . The number of epoch with 9 iterations and the performance (MSE) with 0.0353, gradient decent value of 0.0121 and mu value of 0.01 .
Figure 8 shows the 3 layer neural network training state plot for network with 3 input nodes, 15 hidden nodes and 2 output nodes with combination structure as (3-15-2). The training stops when the validation parameter max fail reached maximum 6 validation checks at epoch 9 with the gradient decent value 0.012087 with reasonable $M u$ value 0.01 which would cause the convergence of the network fast. Because it could be expected that too small $M u$ value would cause the network to converge too slowly. 
Basheer Ahmed Ahmed Ali et al. / American Journal of Applied Sciences 2015, 12 (3): 174.184 DOI: 10.3844/ajassp.2015.174.184

\begin{tabular}{|c|c|c|c|}
\hline \multicolumn{4}{|l|}{ Progress } \\
\hline Epoch: & 0 & 9 iterations & 1000 \\
\hline Performance: & 0.888 & 0.0353 & 0.00 \\
\hline Gradient: & 2.11 & 0.0121 & $1.00 \mathrm{e}-05$ \\
\hline Mu: & 0.00100 & 0.0100 & $1.00 \mathrm{e}+10$ \\
\hline Validation Checks: & 0 & 6 & 6 \\
\hline
\end{tabular}

Fig. 7. Overall progress of the ANN

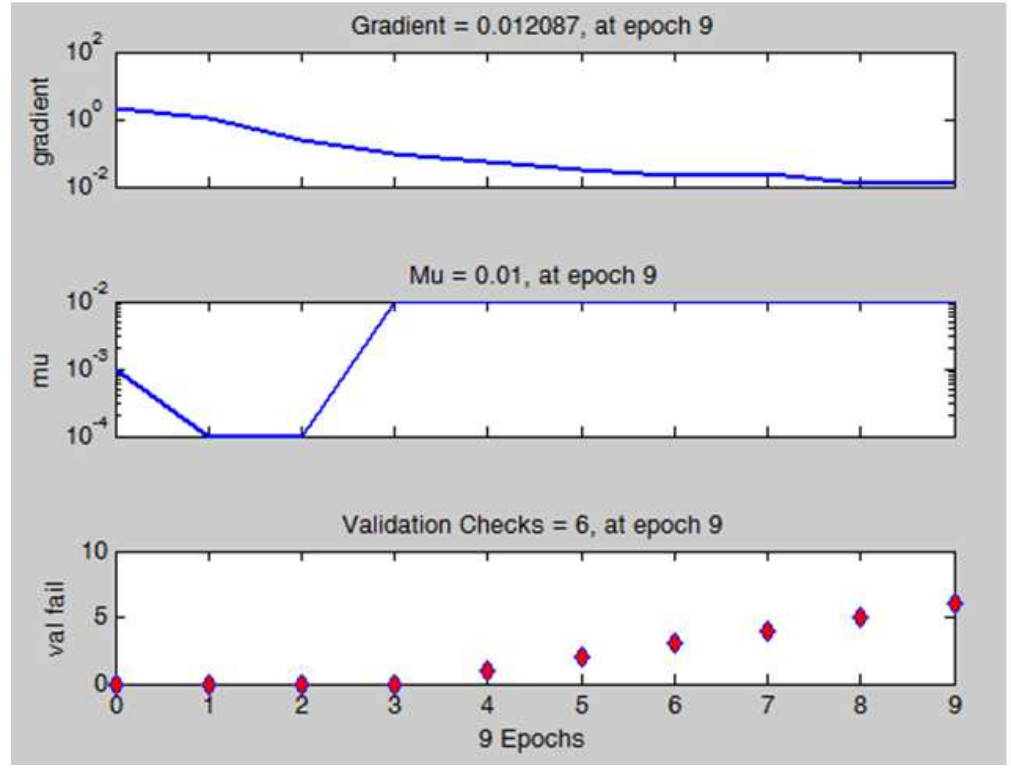

Fig. 8. ANN training state plot for 15 hidden nodes

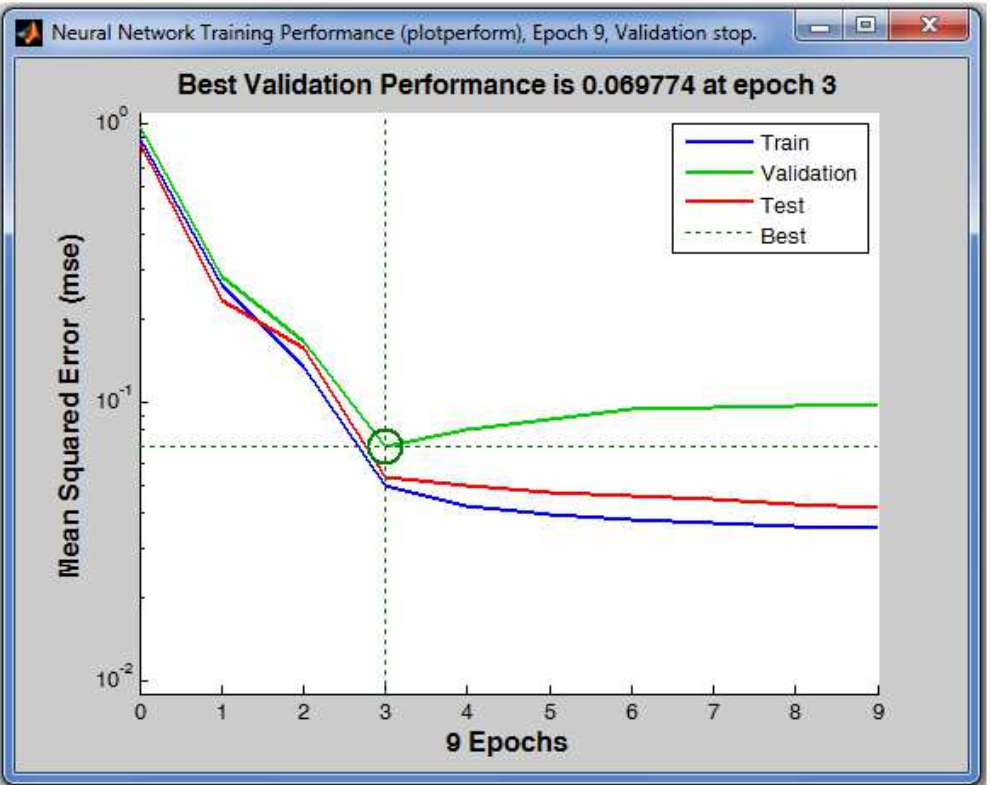

Fig. 9. Network training performance plot 


\section{Network Training Performance}

Neural network training performance plot i.e., plot perform of network is shown in Fig. 9. The value of training, validation and test performances returned by the function train of the network was plotted. The below figure shows that there is no much significant difference between the train and test curves, as they are more or less simillar. This indicates there is no any major problem with the network training. In neural network training if the test curve had increased significantly before the validation curve increased, then there is a possibility that some overfitting might have occured. Even though the training continues until 9 epochs, the best validation performance reached at epoch 3 with the value of 0.069774 .

\section{Regression Analysis}

The regression analysis was performed in ANN model after the network concludes training, testing and validation. It is a statistical process for estimating the relationships between the output and target of the network. The plotregression function takes two parameters (targets, outputs) values and plots the linear regression of targets relative to the outputs. Figure 10 shows the regression plot of training, test, validation and over all regression. The small circle shows the data representation in the model. After the regression plot has been constructed, the diagnosis of the model is important to confirm the goodness of fit. Most commonly used coefficient of determination proof for goodness of fit includes $\mathrm{R}$ squared denoted as $R^{2}$, indicates how well the data points fit a line or curve. In the below figure the best fit was denoted as straight line. The dotted line indicates the outputs equal to targets $(\mathrm{Y}=\mathrm{T})$. The relationship between the outputs and the targets are indicated by the Regression (R) value for every network procedure. If $R=1$, this shows the exact linear Relationship (R) between outputs and targets. The $\mathrm{R}$ value would be rarely perfect in practice. In our model the network shows significantly acceptable $R$ values near to one. The Training $R$ value was 0.89655 , validation $R$ value 0.85545 , test $R$ value 0.88918 and over all $R$ value equals to 0.88944 . This proves the developed model and the network procedure of training, testing and validation are significantly acceptable.

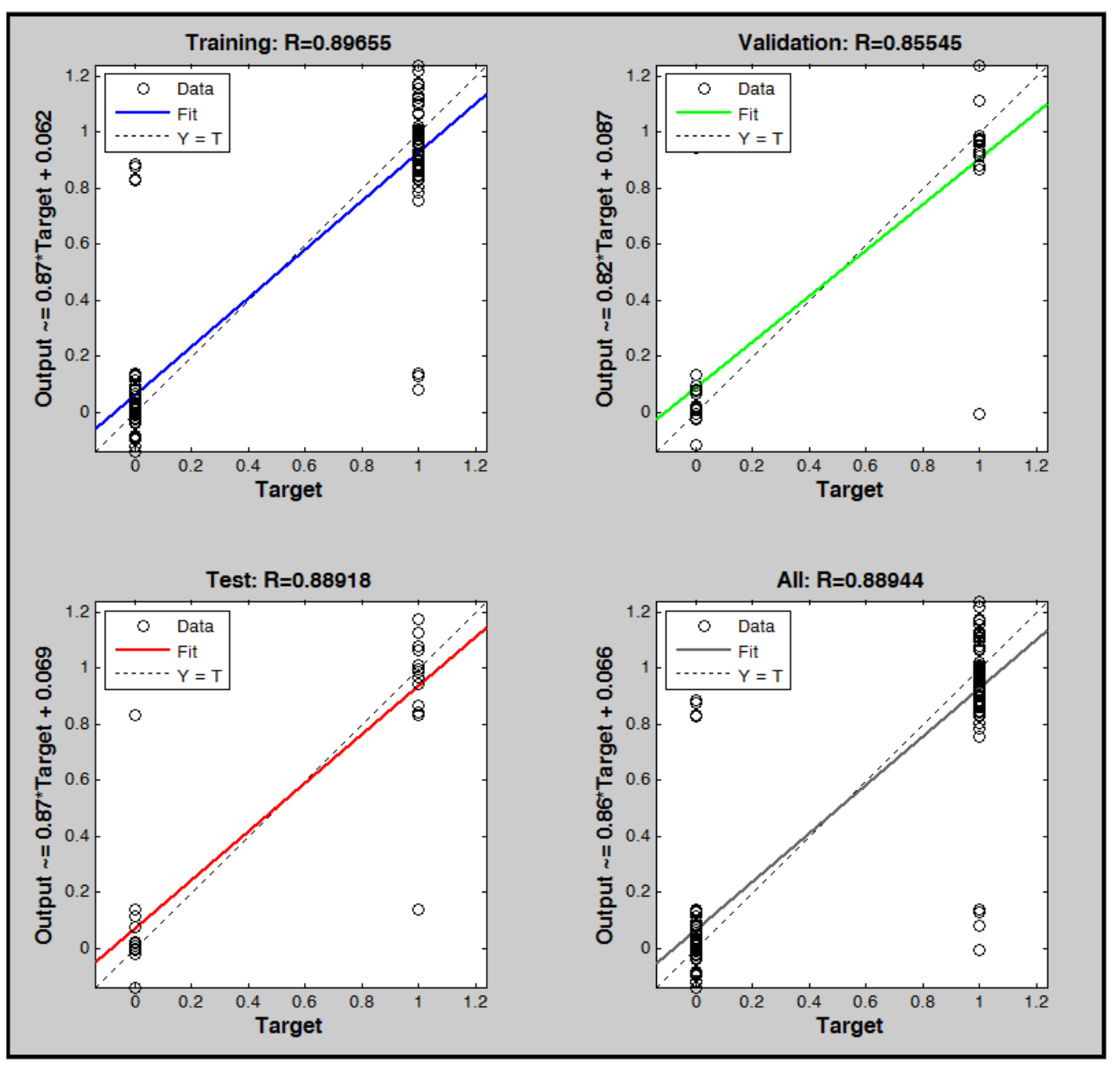

Fig. 10. Regression plot of network 


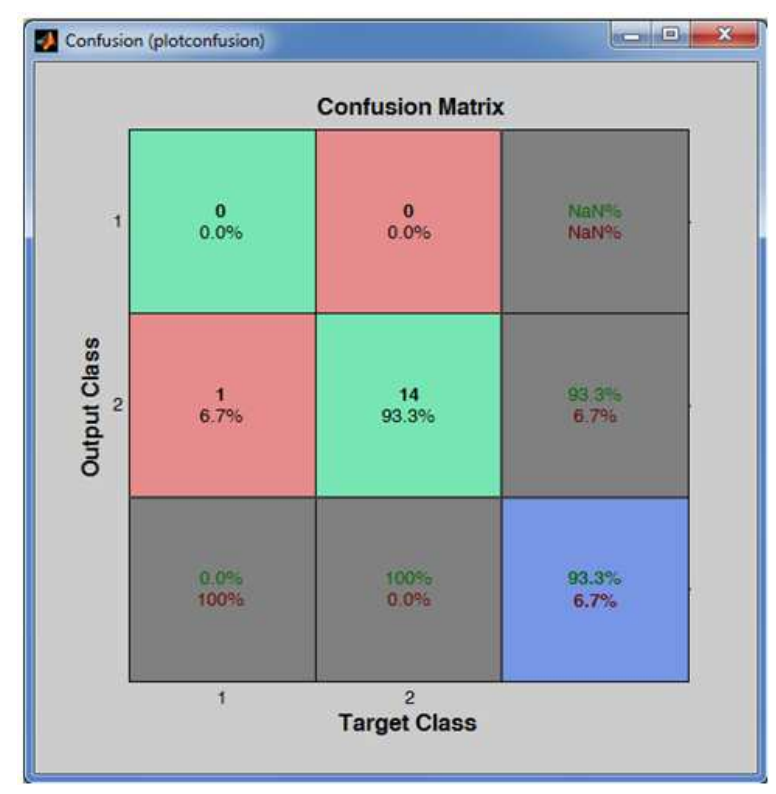

Fig. 11. Confusion matrix

\section{Confusion Matrix}

The confusion matrix or an error matrix also known as contingency table is to identify the accuracy of the classification. In matlab programming, the toolbox neural network can plot confusion matrix. It is a specific table plot which allows the visualization of the performance of Levenberg-Marquardt supervised training algorithm. In this material classification, the system has been trained to classify the optimum material for interior automotive components from natural fibre composites. Ultimately, the below confusion matrix summarize the results of the testing algorithm of the expert neural classifier for further inspection. The classification accuracy and confusion (misclassification) was clearly shown in Fig. 11. The row of the matrix represents the instances in an output class, while each column represents the instances in a target class.

The possible conditions are positive and negative which takes the value true or false. Possible scenarios are true positive, false negative, false positive and true negative. The middle of the diagonal element represents correctly classified observations. So, the percentage of correctly classified was $93.3 \%$, where the remaining $6.7 \%$ can be assumed as incorrectly classified.

Therefore, with all the above observations the artificial neural network model proves to be optimum classifier of materials. Nevertheless how large is the material knowledgebase, a perfect classification could be acheived by the proposed expert neural classifier system.

\section{Conclusion}

This work presents the application of artificial neural network with feed forward network and LevenbergMarquardt training algorithm to classify the natural fibre reinforced composite materials. The classification was made for natural fibre composite materials with high precision. In future the system can be extended with medium and low precision and also guarntees that the ANN is good to integrate with expert system and basically can handle huge amount of data. The ANN with 15 hidden nodes shows best result and the classification accuracy was $93.3 \%$. The research finding proves that ANN is capable to be implemented in materials selection and classification.

\section{Acknowledgement}

The authors would like to thank Ministry of Education, Malaysia for financial support through Exploratory Research Grant Scheme (ERGS) vote number 5527190.

\section{Author's Contributions}

Basheer Ahmed Ahmed Ali: Principal author who conducted the research work, data collection and contributed to the writing of the manuscript.

Mohd Sapuan Salit: Advised with expertise and designed the research plan and approved to complete this manuscript.

Edi Syams Zainudin and Mohamed Othman: Co-supervisor of this research work who provided techinical support for the manuscript.

\section{Ethics}

This manuscript was the original research work carried out by the pricipal author and his supervisory committee. The authors declare that this manuscript was not submitted/accepted elsewhere for publication and free from all ethical issues.

\section{References}

Ahmed Ali, B.A., S.M. Sapuan, E.S. Zainudin and M. Othman, 2013. Java based expert system for selection of natural fibre composite materials. J. Food Agric. Environ., 11: 1871-1877.

Ashby, M.F., 2004. Materials Selection in Mechanical Design. 3rd Edn., Butterworth-Heinemann, Amsterdam, ISBN-10: 0080468640, pp: 624.

Charniya, N.N. and S.V. Dudul, 2011. Classification of material type and its surface properties using digital signal processing techniques and neural networks. Applied Soft Comput., 11: 1108-1116. DOI: $10.1016 /$ j.asoc.2010.02.010

Fazilat, H., M. Ghatarband, S. Mazinani, Z.A. Asadi and M.E. Shiri et al., 2012. Predicting the mechanical properties of glass fiber reinforced polymers via artificial neural network and adaptive neuro-fuzzy inference system. Comput. Mater Sci., 58: 31-37. DOI: 10.1016/j.commatsci.2012.01.012 
Goel, V. and J. Chen, 1996. Application of expert network for material selection in engineering design. Comp Ind., 30: 87-101. DOI: $10.1016 / 0166-3615(96) 00016-4$

Golmohammadi, D., 2011. Neural network application for fuzzy multi-criteria decision making problems. Int. J. Product. Econ., 131: 490-504. DOI: $10.1016 /$ j.ijpe.2011.01.015

Lourakis, M.L.A. and A.A. Argyros, 2005. Is Levenberg-Marquardt the most efficient optimization algorithm for implementing bundle adjustment? Proceedings of the 10th IEEE International Conference on Computer Vision, Oct. 17-21, IEEE Xplore Press, Beijing, pp: 1526-1531. DOI: $10.1109 /$ ICCV.2005.128

Mohanty, A.K., M. Misra, L.T. Drzal, S.E. Selke and B.R. Harte et al., 2005. Natural Fiber Composites in Automotive Applications. In: Natural Fibers, Biopolymers and Biocomposites. Mohanty, A.K., M. Misra, T.L. Drzal (Eds.), CRC Press, ISBN-10: 0203508203, pp: 1-36.

Sapuan, S.M. and H.S. Abdalla, 1998. A prototype knowledge-based system for the material selection of polymeric-based composites for automotive components. Composites Part A: Applied Sci. Manufact., 29: 731-742.

DOI: $10.1016 / \mathrm{S} 1359-835 \mathrm{X}(98) 00049-9$

Sapuan, S.M. and I.M. Mujtaba, 2010. Development of a Prototype Computational Framework for Selection of Natural Fiber Reinforced Polymer Composite Materials Using Neural Network. In: Composite Materials Technology: Neural Network Applications. Sapuan, S.M. and I.M. Mujtaba (Eds.), CRC Press, ISBN-10: 1420093339, pp: 317-340.

Sapuan, S.M., J.Y. Kho, E.S. Zainudin, Z. Leman and B.A. Ahmed Ali et al., 2011. Materials selection for natural fiber reinforced polymer composites using analytical hierarchy process. Ind. J. Eng. Mater. Sci., 18: 255-267.

Sapuan, S.M., M. Harimi and M.A. Maleque, 2003. Mechanical properties of epoxy/coconut shell filler particle composites. Arab. J. Sci. Eng., 28: 171-182.
Smith, L.N., R.M. German and M.L. Smith, 2002. A neural network approach for solution of the inverse problem for selection of powder metallurgy materials. J. Mater. Proc. Technol., 20: 419-425. DOI: $10.1016 / \mathrm{S} 0924-0136(01) 01198-0$

Smith, S.W., 1997. The Scientist and Engineer's Guide to Digital Signal Processing. 1st Edn., California Technical Pub., San Diego, ISBN-10: 0966017633, pp: 626.

Somkuwar, A.K., H.K. Khaira and V. Somkuwar, 2010. Materials selection for product design using artificial neural network technique. J. Eng. Sci. Manag. Edu., 1: 51-54.

Somkuwar, V., J.L. Bhagoriya and H.K. Khaira, 2011. An expert system for aid in material selection process using artificial neural network. Int. J. Adv. Eng. Applic., 3: 169-171.

Sumathi, S. and P. Surekha, 2010. Computational Intelligence Paradigms: Theory and Applications using Matlab, 1st Edn., CRC Press, ISBN-10: 1439809038, pp: 851.

Tan, M.H.A., F. Mat, I.M.A. Rahim, N.L.T. Lile and S. Yaacob, 2011. Classification of materials by modal analysis and neural network. Proceedings of the 5th International Conference on IT and Multimedia, Nov. 14-16, IEEE Xplore Press, Kuala Lumpur, pp: 1-5. DOI: 10.1109/ICIMU.2011.6122753

Trzaska, J. and L.A. Dobrzanski, 2006. Application of neural networks for selection of steel with the assumed hardness after cooling from the austenitising temperature. J. Achieve. Mater. Manuf. Eng., 16: 145-150.

Yegnanarayana, B., 2009. Artificial Neural Networks. PHI Learning Pvt. Ltd., New Delhi, ISBN-10: 8120312538, pp: 476.

Zhou, C., G. Yin and X. Hu, 2009. Multi-objective optimization of material selection for sustainable products: Artificial neural networks and genetic algorithm approach. Mater. Design, 30: 1209-1215. DOI: $10.1016 /$ j.matdes.2008.06.006 